\title{
The relationship of Beck depression inventory with vitamin D levels and visceral fat mass in cancer patients
}

Savas Tuna, Meral Mert, Ozlem Soyluk*, Yildiz Okuturlar, Hakan Kocoglu, Didem Tastekin*

Bakirkoy Dr. Sadi Konuk Education and Research Hospital

Istanbul University, Faculty of Medicine

\section{Objectives:}

The aim of this study is to determine the relationship of Beck Depression Inventory (BDI) with vitamin $\mathrm{D}$ levels and total and visceral fat mass in cancer patients.

\section{Methods:}

A total of 219 patients were included in the study. All patients underwent blood tests including prealbumin, vitamin D. Also patients' BMI values were calculated and total and visceral fat masses of all patients were measured by bio-impedance analyzer. All subjects completed a self-administered BDI questionnaire.

\section{Results:}

A total of 219 patients consisted of $53.9 \%(\mathrm{n}=118)$ female and $46.1 \%(\mathrm{n}=101)$ male patients with median age $52.41 \pm 13.66$ (range, 19 - 84) years. Mean BMI value was $24.70 \pm 3.75 \mathrm{~kg} / \mathrm{m} 2$; mean BDI score was $13.02 \pm 8.72$; and mean prealbumin level was $0.21 \pm 0.07 \mathrm{~g} / \mathrm{L}$. BMI was negatively correlated with BDI both in all study group $(\mathrm{p}=0.002)$ and in male patients $(\mathrm{p}=0.011)$ whereas there was no correlation in female patients between BMI and BDI $(\mathrm{p}=0.237)$. Prealbumin levels, vitamin D levels and hemoglobin levels were negatively correlated with BDI ( $p<0.05$ for all). But no significant correlation was determined between total fat mass, visceral fat mass levels and BDI ( $p>0.05)$. Depression status had a significant relationship with BMI, vitamin D levels, prealbumin levels and malnutrition status in patients $(p=0.008$, $0.001,0.001$, and 0.001 , respectively).

\section{Conclusion:}

We have determined a significant correlation between vitamin D levels and BDI scores which indicate depression status in cancer patients. There was no correlation between BDI and visceral fat mass. There was a negative correlation between BDI score and BMI levels but this was not associated with total or visceral fat mass in cancer patients. Thus vitamin D levels could be used to determine the depression and nutritional statuses which might help to improve the clinical outcomes in cancer patients. 